\title{
Deep SDSS optical spectroscopy of distant halo stars
}

\section{Chemical analysis of extremely metal-poor stars ${ }^{\star}$}

\author{
E. Fernández-Alvar ${ }^{1}$, C. Allende Prieto ${ }^{2,3}$, T. C. Beers ${ }^{4}$, Y. S. Lee ${ }^{5}$, T. Masseron ${ }^{6}$, and D. P. Schneider ${ }^{7}$ \\ 1 Instituto de Astronomía, Universidad Nacional Autónoma de Mexico, AP 70-264, 04510 Ciudad de México, Mexico \\ e-mail: emma@astro.unam.mx \\ 2 Instituto de Astrofísica de Canarias, Vía Láctea, 38205 La Laguna, Tenerife, Spain \\ 3 Universidad de La Laguna, Departamento de Astrofísica, 38206 La Laguna, Tenerife, Spain \\ 4 Department of Physics and JINA Center for the Evolution of the Elements, University of Notre Dame, Notre Dame, IN 46556, \\ USA \\ 5 Department of Astronomy and Space Science, Chungnam National University, 99 Daehak-ro, 34134 Daejeon, Republic of Korea \\ 6 Institute of Astronomy, University of Cambridge, Madingley Road, CB3 0HA, Cambridge, UK \\ 7 Department of Astronomy and Astrophysics, The Pennsylvania State University, University Park, PA 16802, USA
}

Received 3 May 2016 / Accepted 15 June 2016

\begin{abstract}
Aims. We present the results of an analysis of 107 extremely metal-poor (EMP) stars with metallicities lower than $[\mathrm{Fe} / \mathrm{H}]=-3.0$, identified in medium-resolution spectra in the Sloan Digital Sky Survey (SDSS). Our analysis provides estimates of the stellar effective temperatures and surface gravities, as well as iron, calcium, and magnesium abundances.

Methods. We followed the same method as in previous papers of this series. The method is based on comparisons of the observed spectra with synthetic spectra. The abundances of $\mathrm{Fe}, \mathrm{Ca}$, and $\mathrm{Mg}$ were determined by fitting spectral regions that are dominated by lines of each element. In addition, we present a technique to determine upper limits for elements whose features are not detected in a given spectrum. We also analyzed our sample with the SEGUE stellar parameter pipeline to obtain additional determinations of the atmospheric parameters and iron and alpha-element abundances, which we thend compare with ours. In addition, we used these parameters to infer $[\mathrm{C} / \mathrm{Fe}]$ ratios.

Results. Ca is typically the only element in these spectra with a moderate to low signal-to-noise ratio and medium resolution in this metallicity regime with lines that are sufficiently strong to reliably measure its abundance. Fe and $\mathrm{Mg}$ exhibit weaker features that in most cases only provide upper limits. We measured $[\mathrm{Ca} / \mathrm{Fe}]$ and $[\mathrm{Mg} / \mathrm{Fe}]$ for EMP stars in the SDSS spectra and conclude that most of the stars exhibit the typical enhancement level for $\alpha$-elements, $\sim+0.4$, although some stars for which only $[\mathrm{Fe} / \mathrm{H}]$ upper limits could be estimated indicate higher $[\alpha / \mathrm{Fe}]$ ratios. We also find that $26 \%$ of the stars in our sample can be classified as carbon-enhanced metalpoor (CEMP) stars and that the frequency of CEMP stars also increases with decreasing metallicity, as has been reported for previous samples. We identify a rare, bright $(g=11.90)$ EMP star, SDSS J134144.61+474128.6, with $[\mathrm{Fe} / \mathrm{H}]=-3.27,[\mathrm{C} / \mathrm{Fe}]=+0.95$, and elevated magnesium $([\mathrm{Mg} / \mathrm{Fe}]=+0.62)$, an abundance pattern typical of CEMP-no stars.
\end{abstract}

Key words. stars: abundances - stars: Population III - methods: data analysis - catalogs - Galaxy: halo - stars: carbon

\section{Introduction}

Very metal-poor (VMP; $[\mathrm{Fe} / \mathrm{H}]<-2.0)$ and extremely metalpoor $(\mathrm{EMP} ;[\mathrm{Fe} / \mathrm{H}]<-3.0)$ stars provide the opportunity of deepening our understanding of the early chemical evolution of the Milky Way and the Universe. In most cases, their atmospheres exhibit the chemical compositions of the gas from which they formed, enriched by the nucleosynthetic yields of the first stellar populations. In addition, recent investigations of the Milky Way's stellar halo have revealed that it is not a homogeneous entity, but instead comprises multiple populations (Carollo et al. 2007, 2010; de Jong et al. 2010; Nissen \& Schuster 2010, 2011; Beers et al. 2012; An et al. 2013, 2015; Allende Prieto et al. 2014; Chen et al. 2014; Janesh et al. 2016). VMP and EMP stars are therefore useful probes of the assembly of the Galaxy as well.

\footnotetext{
* Tables 4-6 are only available at the CDS via anonymous ftp to cdsarc.u-strasbg. fr (130.79.128.5) or via http://cdsarc.u-strasbg.fr/viz-bin/qcat?J/A+A/593/A28
}

Early survey work, for example, the HK survey of Beers and colleagues (Beers et al. 1985, 1992) and the Hamburg/ESO survey of Christlieb and collaborators (Reimers \& Wisotzki 1997; Christlieb 2003), provided the first large lists of several thousand VMP and EMP stars. More recently, advances in astronomical instrumentation have enabled multiplexed spectroscopy of even larger numbers of (generally fainter) stars, such as the Sloan Digital Sky Survey (SDSS; York et al. 2000); this produced samples of VMP and EMP stars that cover a wide range of distances from the Sun.

High-resolution spectroscopic follow-up of these targets has provided abundance measurements for numerous elements, revealing the existence of chemical peculiarities that have greatly expanded our knowledge of the different nucleosynthetic pathways that contributed to the early chemical evolution of the Galaxy (e.g., Cayrel et al. 2004; Arnone et al. 2005; Aoki et al. 2005, 2013; Cohen et al. 2004, 2007, 2008; Bonifacio et al. 2009; Lai et al. 2009; Roederer 2009; Roederer et al. 2014). Until recently, relatively few stars had confirmed metallicities of 
$[\mathrm{Fe} / \mathrm{H}]<-3.0$, making it difficult to infer the global characteristics of EMP stars. Since 2005, more than 200 stars with $[\mathrm{Fe} / \mathrm{H}]<-3.0$ have been confirmed based on high-resolution spectroscopic analyses (e.g., Barklem et al. 2005; Caffau et al. 2013a,b; Yong et al. 2013), including more than 50 stars with $[\mathrm{Fe} / \mathrm{H}]<-3.5$, and over 20 with $[\mathrm{Fe} / \mathrm{H}]<-4.0$ (Barklem et al. 2005; Frebel et al. 2005, 2015; Cohen et al. 2008; Caffau et al. 2011a,b, 2012, 2013b; Bonifacio et al. 2012; Aoki et al. 2013; Smolinski et al. 2011; Yong et al. 2013; Keller et al. 2014; Allende Prieto et al. 2015; Li et al. 2015; Placco et al. 2015; Meléndez et al. 2016).

The SDSS database comprises over 900000 stellar spectra and is now the dominant source of confirmed EMP stars. However, the relatively low resolution $(R \sim 2000)$ and limited signalto-noise ratio $(S / N \sim 30-40)$ of the SDSS spectra themselves make it difficult to derive elemental abundances at very low metallicities.

This paper is the third in a series devoted to analyses of Milky Way halo stars based on the low-resolution SDSS spectroscopic data. We present 107 stars with metallicities $[\mathrm{Fe} / \mathrm{H}]<$ -3.0 for which the stellar atmospheric parameters and chemical abundances of $[\mathrm{Fe} / \mathrm{H}],[\mathrm{Mg} / \mathrm{H}]$, and $[\mathrm{Ca} / \mathrm{H}]$ have been estimated. We also report on a method for estimating upper limits for these abundances from the SDSS spectra. This technique enables determining the lowest required $\mathrm{S} / \mathrm{N}$ for estimating the abundance for a given element as a function of effective temperature $\left(T_{\text {eff }}\right)$ and surface gravity $(\log g)$.

This paper is outlined as follows. In Sect. 2 we briefly discuss the data used in the analysis, which is described in Sect. 3. In this section we also introduce our method for estimating upper limits. Section 4 reports on a comparison with estimates from other analyses, including high-resolution spectroscopy. The results are summarized in Sect. 5. Finally, we present our conclusions and a brief discussion in Sect. 6.

\section{Observations}

Our stellar spectra come from the SDSS. This project, started in 2000, is now on its third extension, SDSS-IV (Alam et al. 2015), and comprises a set of surveys devoted to a variety of areas, from cosmology to the evolution of galaxies and the Milky Way to the search for extrasolar planets. Its first extension, SDSS-II, included a specific stellar project, the Sloan Extension for Galactic Understanding and Exploration (SEGUE; Yanny et al. 2009), directed at investigating the structure, formation, and chemical evolution of the Galaxy. SEGUE-2, a subsurvey of SDSS-III (Eisenstein et al. 2011), increased the number of stellar spectra and focused on observing distant halo stars.

SEGUE, SEGUE-2, and other SDSS programs (including the main SDSS galaxy redshift survey and BOSS, the Baryon Oscillations Spectroscopic Survey, see Dawson et al. 2013) obtain spectra for color-selected samples of F-type main-sequence turn-off stars observed for calibration purposes. Calibration stars taken during the main survey and BOSS have the advantage of being located across the entire SDSS footprint at high Galactic latitudes (instead of the limited number of directions probed by SEGUE and SEGUE-2) and include halo stars at distances of up to $\sim 100 \mathrm{kpc}$.

The spectra were obtained with a pair of spectrographs on the SDSS $2.5 \mathrm{~m}$ telescope (Gunn et al. 2006; Smee et al. 2013) at Apache Point Observatory, with a wavelength-dependent resolving power of $1300<R<3000$ for the spectral range $\sim 3800<\lambda<9000 \AA$. The spectrographs were updated before the beginning of BOSS observations to increase their efficiency and spectral range (to $3600<\lambda<10000 \AA$ ). Additional details on these spectra can be found in the technical papers of the surveys (York et al. 2000; Yanny et al. 2009; Dawson et al. 2013; Alam et al. 2015), as well as in previous papers in this series (Allende Prieto et al. 2014; Fernández-Alvar et al. 2015; hereafter Papers I and II).

\section{Analysis}

\subsection{Measurement of stellar parameters and chemical abundances}

We wish to estimate stellar atmospheric parameters and individual element abundances for a sample of extremely metal-poor stars belonging to the halo system. Following the same strategy as described in Papers I and II, we made use of the FERRE code (Allende Prieto et al. 2006) to constrain the stellar atmospheric parameters: effective temperature, $T_{\mathrm{eff}}$, surface gravity, $\log g$, and the global metallicity, $[\mathrm{M} / \mathrm{H}]$. We adopted the notation $[\mathrm{X} / \mathrm{H}]=$ $\log _{10}\left(\frac{N(\mathrm{X})}{N(\mathrm{H})}\right)-\log _{10}\left(\frac{N(\mathrm{X})}{N(\mathrm{H})}\right)$, where $\mathrm{X}$ is any chemical element, $N(\mathrm{X})$ is the number density of the nuclei of this element, and $N(\mathrm{H})$ is the number density of hydrogen nuclei. $[\mathrm{M} / \mathrm{H}]$ is the iron abundance determined from fitting the available spectral range, which includes spectral features from other metals.

The search was performed by comparison with a grid of synthetic spectra covering a wide range of parameter space, seeking the minimum $\chi^{2}$ using quadratic Bezier interpolation between the model spectra (Auer 2003). We employed the same three-dimensional spectral library $\left(T_{\text {eff }}, \log g\right.$, and $\left.[\mathrm{M} / \mathrm{H}]\right)$ as in Paper I, calculated from one-dimensional plane-parallel Kurucz model atmospheres (Castelli \& Kurucz 2003), which consider local thermodynamical equilibrium (LTE). The grid covers the ranges $4750<T_{\text {eff }}<6500 \mathrm{~K}, 0.5<\log g<4.5$, and $-5.0<$ $[\mathrm{M} / \mathrm{H}]<+0.5$, in steps of $250 \mathrm{~K}, 0.5 \mathrm{dex}$, and $0.5 \mathrm{dex}$, respectively. More details of the model atmospheres, line data, and the opacities used in the generation of the spectral library can be found in Paper I.

The FERRE routine enables searches for one, several, or all of the atmospheric parameters in a library of synthetic spectra. When the atmospheric parameters were determined, we searched for a limited set of elemental abundances, holding the atmospheric parameters fixed from the analysis of the full spectrum. In our model grids we varied the abundances of all metals relative to hydrogen in solar proportions (with the exception of the $\alpha$-elements, which are enhanced by +0.4 dex for metal-poor stars). Searching for $[\mathrm{M} / \mathrm{H}]$, but restricting the fit to regions dominated by individual lines of other elements, enables estimating the abundance of each of these elements, corresponding to the value of $[\mathrm{M} / \mathrm{H}]$ that best reproduces the shapes of their associated lines. A more detailed explanation can be found in Paper II.

We first estimated the stellar parameters using the full spectral range provided by the observations. The spectral range was limited to $3850<\lambda<9190 \AA$ to ensure consistency with our previous analysis in Papers I and II, which examined the BOSS spectra and those from earlier SDSS/SEGUE observations. From this analysis we selected our primary targets to have metallicities in the range $-4.0<[\mathrm{M} / \mathrm{H}]<-3.0$. We rejected spectra that appeared to be double-lined binaries or white dwarfs. The $\mathrm{S} / \mathrm{N}$ (calculated as the median value per pixel in the range $4885<\lambda<5500 \AA$ for each spectrum) varied between $20<S / N<90$; the derived values of $T_{\text {eff }}$ and $\log g$ varied between $5170<T_{\text {eff }}<6500 \mathrm{~K}$ and $0.5<\log g<4.5$, respectively. Holding $T_{\text {eff }}$ and $\log g$ fixed, we repeated the search in the 
E. Fernández-Alvar et al.: Deep SDSS optical spectroscopy of distant halo stars. III.
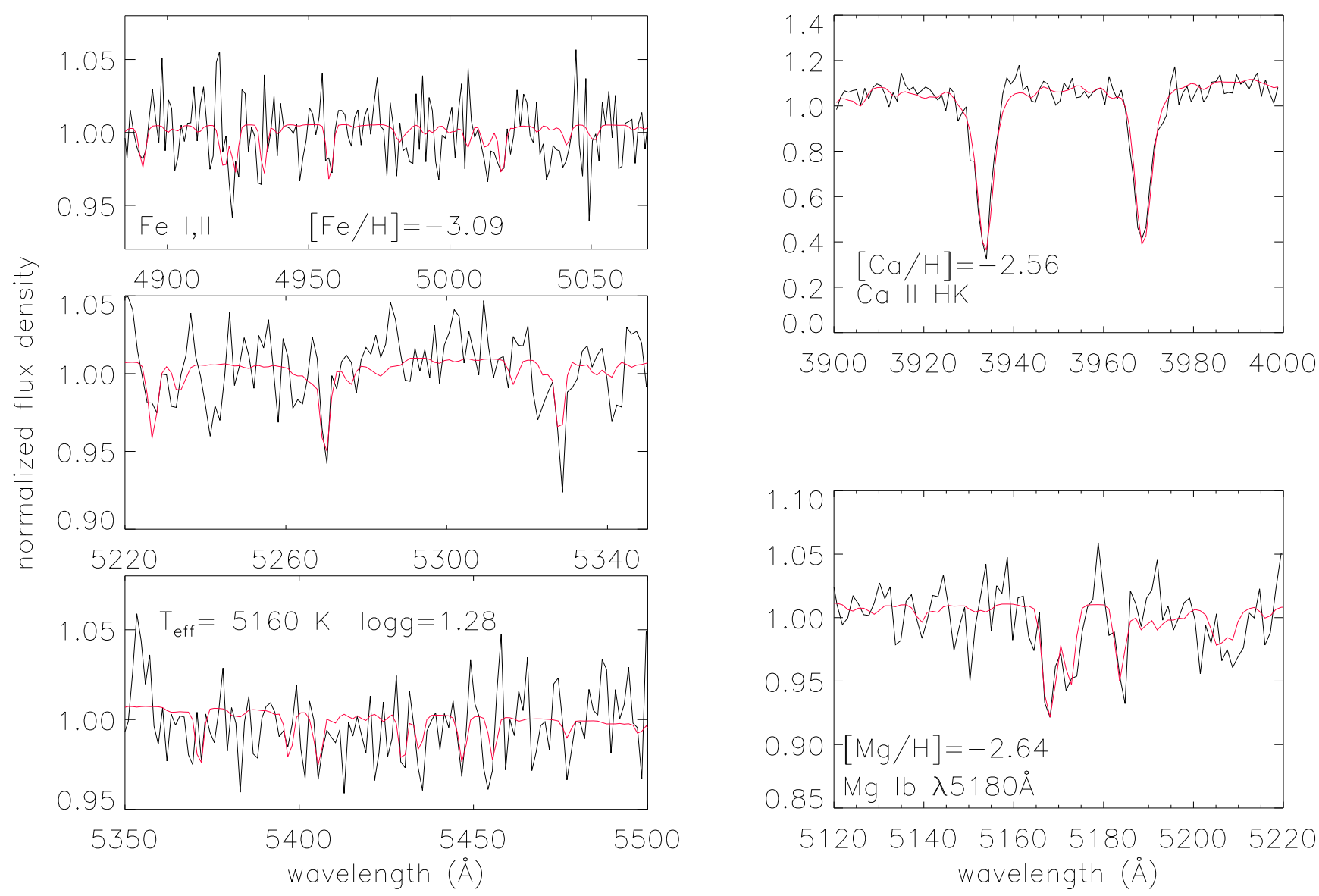

Fig. 1. Model fits for a SDSS/SEGUE star, SDSS J093339.24+310245.4, with the mean S/N of the sample, 40 . The three panels in the left column correspond to the fit regions from which the $[\mathrm{Fe} / \mathrm{H}]$ abundance was determined. The derived iron abundance is shown in the legend of the upper panel, while the temperature and surface gravity estimates are shown in the legend of the lower panel. The panels in the right column show the CaII HK doublet and the MgIb triplet that were fit to determine $\mathrm{Ca}$ and $\mathrm{Mg}$ abundances, with the derived estimates shown in the legends.

$[\mathrm{M} / \mathrm{H}]$ dimension of the grid by fitting selected regions in the spectra that contain atomic lines of $\mathrm{Fe}, \mathrm{Ca}$, or $\mathrm{Mg}$.

In this analysis we fit the following spectral ranges (shown in Fig. 1): $4885<\lambda<5070 \AA, 5220<\lambda<5280 \AA$, and $5295<$ $\lambda<5500 \AA$ to determine Fe abundances; $5160<\lambda<5190 \AA$ (the Mg Ib triplet) for Mg abundances; and $3910<\lambda<3990 \AA$ (the $\mathrm{Ca}$ II $\mathrm{H}$ and $\mathrm{K}$ resonance doublet) for $\mathrm{Ca}$ abundances. These are the regions with the highest sensitivity in the optical spectral range for each element. The spectra were normalized by splitting them into $100 \AA$ pieces ( $200 \AA$ for the Fe I window between 4875 and $5510 \AA$ ) and dividing each piece by its mean flux value. Our synthetic spectra were treated in the same manner. From the new metallicity estimates, we obtained the abundance values of $[\mathrm{Mg} / \mathrm{H}]$ and $[\mathrm{Ca} / \mathrm{H}]([\mathrm{Fe} / \mathrm{H}]$ is straightforward $)$, considering the relation with $[\mathrm{Fe} / \mathrm{H}]$ adopted in the construction of the spectral grid.

\subsection{Method for estimating upper limits}

The relatively low $\mathrm{S} / \mathrm{N}$ and modest resolution of our data complicate the estimation of some elemental abundances, in particular for $\mathrm{Fe}$ and $\mathrm{Mg}$, whose line detections become marginal for low metallicities at the $\mathrm{S} / \mathrm{N}$ of our spectra. For this reason, we developed a method for determining upper limits on the abundance of an element as a function of $\mathrm{S} / \mathrm{N}, T_{\mathrm{eff}}, \log g$, and metallicity, $[\mathrm{M} / \mathrm{H}]$.

We simulated the observed spectra by smoothing our spectral library to $R=2000$ and adding Gaussian noise. From a simulated spectrum with a particular $\mathrm{S} / \mathrm{N}, T_{\text {eff }}, \log g$, and $[\mathrm{M} / \mathrm{H}]$ (hereafter $\left.[\mathrm{M} / \mathrm{H}]_{0}\right)$, we evaluated the $\chi^{2}$ and its error $\left(\sigma=\sqrt{4 \chi^{2}}\right)$, comparing with noise-free spectra over $-5.0<[\mathrm{M} / \mathrm{H}]<-2.5$ for the same spectral windows as were used to determine the abundances of $\mathrm{Fe}, \mathrm{Mg}$, and $\mathrm{Ca}$. We calculated the slope of the $\chi^{2}$ curve in the range $-5.0<[\mathrm{M} / \mathrm{H}]<[\mathrm{M} / \mathrm{H}]_{0}$. Figure 2 illustrates the method. Repeating this process at different $\mathrm{S} / \mathrm{Ns}$, from 20 to 90 (in steps of 5), we defined the minimum S/N (hereafter $\mathrm{S} / \mathrm{N}_{0}$ ) at which the slope becomes significant (i.e., its error is lower than its value) for each $[\mathrm{M} / \mathrm{H}]_{0}$. This calculation was performed a hundred times to statistically refine this lowest required $\mathrm{S} / \mathrm{N}_{0}$ value.

By evaluating simulations at $T_{\text {eff }}$ values from 4750 to $6500 \mathrm{~K}$ in steps of $250 \mathrm{~K}$, two $\log g$ values, 1.5 and 4.2, and metallicities from -4.8 to -2.5 in steps of 0.1 dex, we established the lowest required $\mathrm{S} / \mathrm{N}_{0}$ vs. $[\mathrm{Fe} / \mathrm{H}]_{0}$ curves for each combination of stellar atmospheric parameters. The $\mathrm{S} / \mathrm{N}$ range considered was $20-90$ for Fe and $\mathrm{Mg}$. We extended the lower limit to $S / N=5$ in the Ca evaluation, since the $\mathrm{S} / \mathrm{N}$ over $3900<\lambda<4000 \AA$ in the analyzed spectra is lower than 20 in some cases. These curves were used to evaluate the reliability of our $\mathrm{Fe}, \mathrm{Mg}$, and $\mathrm{Ca}$ abundance determinations. Figure 3 shows the results for $\log g=1.5$ and 


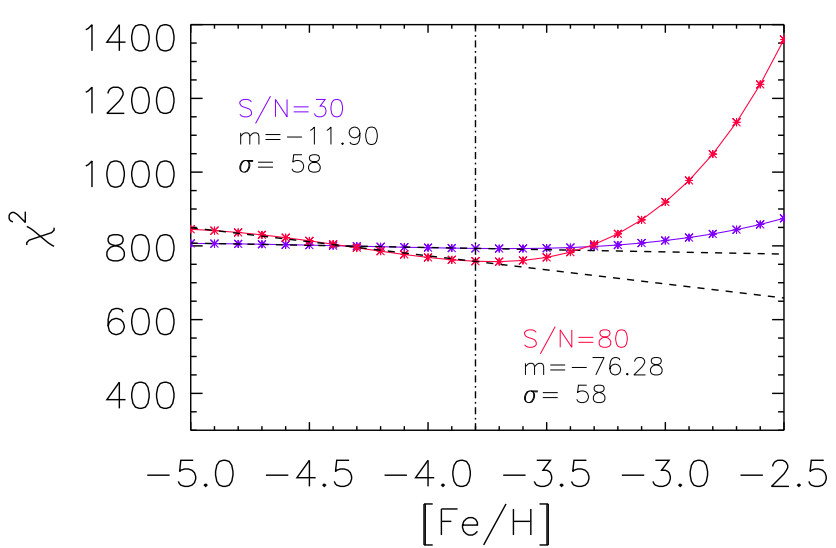

Fig. 2. $\chi^{2}$ obtained from the evaluation of a simulated spectrum at $[\mathrm{M} / \mathrm{H}]_{0}=[\mathrm{Fe} / \mathrm{H}]_{0}=-3.8$ (marked with a vertical dotted line) compared with synthetic spectra over a range of metallicities $[\mathrm{M} / \mathrm{H}]$ (from -5.0 to -2.5 with a step of $0.1 \mathrm{dex}$ ). The blue line shows the resulting $\chi^{2}$ curve for a simulation with $S / N=30$, and the red line applies to $S / N=80$. The two black dotted lines indicate the linear fits up to the $[\mathrm{M} / \mathrm{H}]_{0}$ corresponding to the minimum $\chi^{2}$, from which the slope and its uncertainty are derived.

4.2. We accepted a given abundance determination when it was higher than the corresponding $\mathrm{S} / \mathrm{N}$ at which the modulus of the difference with the $\mathrm{S} / \mathrm{N}$ of the observation is smallest. Otherwise, when the value determined by FERRE was lower than this limit, we considered the estimate as an upper limit for the abundance.

This method can be used for the analysis of spectra with a low resolution and low $\mathrm{S} / \mathrm{N}$. From Fig. 3 we can infer that $T_{\text {eff }}$ affects the abundance determination of the three elements more strongly than $\log g$, as expected.

Iron is the most difficult to measure. Although more lines are available than for the other two elements, their weakness in this metallicity regime makes them challenging to measure in the presence of noise. Our calculations indicate that a $S / N>90$ is necessary to reliably determine $[\mathrm{Fe} / \mathrm{H}] \leq-4.2$ over the $T_{\text {eff }}$ and $\log g$ ranges considered and our selected spectral range. At $S / N \sim 90$, the upper limit changes by $\sim 1$ dex, from -4.0 to -3.0 , between 4750 to $6500 \mathrm{~K}$ at $\log g=1.5$ (becoming larger as $\log g$ increases). By contrast, the calcium abundance can be measured down to $[\mathrm{Ca} / \mathrm{H}] \sim-4.2$ for spectra with a median $S / N \sim 10$ for stars with $T_{\text {eff }}=4750 \mathrm{~K}$ and $\log g=4.2$.

To provide a convenient tool for estimating the lowest required $\mathrm{S} / \mathrm{N}$ for measuring the abundances of these elements from the spectral windows considered in this work, we derived an analytic function that fits the curves obtained from the simulations. We found that these curves can be well-reproduced by a secondorder polynomial (a convex parabola) and a straight line through the vertex of the parabola,

$$
\mathrm{S} / \mathrm{N}= \begin{cases}a\left(T_{\mathrm{eff}}\right)[\mathrm{X} / \mathrm{H}]^{2}+b\left(T_{\mathrm{eff}}\right)[\mathrm{X} / \mathrm{H}]+c\left(T_{\mathrm{eff}}\right) & \text { if }[\mathrm{X} / \mathrm{H}]<\frac{-b}{2 a} \\ d\left(T_{\mathrm{eff}}\right)[\mathrm{X} / \mathrm{H}]+e\left(T_{\mathrm{eff}}\right) & \text { if }[\mathrm{X} / \mathrm{H}]>\frac{-b}{2 a}\end{cases}
$$

We modeled the dependence of the polynomial coefficients with $T_{\text {eff }}$ as second-order polynomials. The applicability domain for each second-order polynomial is defined below and above certain $T_{\text {eff }}$ values $(5500 \mathrm{~K}$ at $\log g=1.5$ and $6000 \mathrm{~K}$ at $\log g=4.2)$.

$$
\begin{aligned}
& a\left(T_{\text {eff }}\right)=p_{11} T_{\text {eff }}^{2}+p_{12} T_{\text {eff }}+p_{13} \\
& b\left(T_{\text {eff }}\right)=p_{21} T_{\text {eff }}^{2}+p_{22} T_{\text {eff }}+p_{23} \\
& c\left(T_{\text {eff }}\right)=p_{31} T_{\text {eff }}^{2}+p_{32} T_{\text {eff }}+p_{33} .
\end{aligned}
$$

\begin{tabular}{|c|c|c|c|}
\hline \multicolumn{4}{|c|}{$\log g=1.5$} \\
\hline \multicolumn{4}{|c|}{$T_{\text {eff }}<5500 \mathrm{~K}$} \\
\hline$p_{1}$ & $-1.122267 \mathrm{e}-04$ & $1.116517 \mathrm{e}+00$ & $-2.725528 \mathrm{e}+03$ \\
\hline$p_{2}$ & $-7.752576 \mathrm{e}-04$ & $7.661329 \mathrm{e}+00$ & $-1.862050 \mathrm{e}+04$ \\
\hline$p_{3}$ & $-1.307124 \mathrm{e}-03$ & $1.285527 \mathrm{e}+01$ & $-3.112059 \mathrm{e}+04$ \\
\hline \multicolumn{4}{|c|}{$T_{\text {eff }}>5500 \mathrm{~K}$} \\
\hline$p_{1}$ & $-4.702741 \mathrm{e}-05$ & $5.608045 \mathrm{e}-01$ & $-1.619006 \mathrm{e}+03$ \\
\hline$p_{2}$ & $-2.673086 e-04$ & $3.143082 \mathrm{e}+00$ & $-8.982544 \mathrm{e}+03$ \\
\hline$p_{3}$ & $-3.688757 \mathrm{e}-04$ & $4.301038 \mathrm{e}+00$ & $-1.219482 \mathrm{e}+04$ \\
\hline$l_{1}$ & $-1.493794 \mathrm{e}-02$ & $6.539818 \mathrm{e}+01$ & \\
\hline$l_{2}$ & $-3.479122 \mathrm{e}-02$ & $1.706138 \mathrm{e}+02$ & \\
\hline \multicolumn{4}{|c|}{$\log g=4.2$} \\
\hline \multicolumn{4}{|c|}{$T_{\text {eff }}<6000 \mathrm{~K}$} \\
\hline$p_{1}$ & $6.350261 \mathrm{e}-05$ & $-6.807567 \mathrm{e}-01$ & $1.868088 \mathrm{e}+03$ \\
\hline$p_{2}$ & $4.025916 \mathrm{e}-04$ & $-4.391630 \mathrm{e}+00$ & $1.219878 \mathrm{e}+04$ \\
\hline$p_{3}$ & $6.643223 e-04$ & $-7.321549 \mathrm{e}+00$ & $2.047375 e+04$ \\
\hline \multicolumn{4}{|c|}{$T_{\text {eff }}>6000 \mathrm{~K}$} \\
\hline$p_{1}$ & $4.131073 e-04$ & $-4.953010 \mathrm{e}+00$ & $1.490776 \mathrm{e}+04$ \\
\hline$p_{2}$ & $2.245832 \mathrm{e}-03$ & $-2.701466 e+01$ & $8.152300 \mathrm{e}+04$ \\
\hline$p_{3}$ & $3.071746 \mathrm{e}-03$ & $-3.703024 \mathrm{e}+01$ & $1.119596 \mathrm{e}+05$ \\
\hline$l_{1}$ & $-5.311476 \mathrm{e}-03$ & $1.899590 \mathrm{e}+01$ & \\
\hline$l_{2}$ & $-7.989891 \mathrm{e}-03$ & $3.985540 \mathrm{e}+01$ & \\
\hline
\end{tabular}

Table 1. Second-order polynomial coefficients that reproduce the parabolic and linear polynomial parameters as a function of $T_{\text {eff }}$ for each of the $\log g$ values considered, 1.5 and 4.2, to estimate Fe.

A single first-order polynomial fits the dependence of the other two coefficients on $T_{\text {eff }}$ well,

$d\left(T_{\text {eff }}\right)=l_{11} T_{\text {eff }}+l_{12}$

$e\left(T_{\text {eff }}\right)=l_{21} T_{\text {eff }}+l_{22}$.

Tables 1-3 show the coefficients of these polynomials for each element for the two $\log g$ values evaluated, 1.5 and 4.2.

\section{Verification with other analyses}

\subsection{Comparison with the SSPP analysis}

The SEGUE stellar parameter pipeline (SSPP) was developed to analyze the stellar spectra gathered in the SDSS/SEGUE surveys (see Lee et al. 2008a,b; Allende Prieto et al. 2008; Smolinski et al. 2011; Lee et al. 2011 for details) and can be used to estimate $T_{\text {eff }}, \log g,[\mathrm{Fe} / \mathrm{H}]$, and $[\alpha / \mathrm{Fe}]-$ the $[\alpha / \mathrm{Fe}]$ corresponds to the $[\mathrm{Mg} / \mathrm{Fe}],[\mathrm{Si} / \mathrm{Fe}],[\mathrm{Ca} / \mathrm{Fe}]$, and $[\mathrm{Ti} / \mathrm{Fe}]$ global measurement from the spectral range 4500-5500 $\AA$ fit to the observational data with synthetic spectra. Recently, the SSPP has been extended to be capable of estimating $[\mathrm{C} / \mathrm{Fe}]$ as well (Lee et al. 2013). For the sake of comparison, we also evaluated our sample of moderate-resolution SDSS spectra with this tool.

We compared the stellar parameters and abundance measurements obtained with the SSPP with our present analysis results. Our analysis returns a lower estimated $T_{\text {eff }}$ than the SSPP $(\delta=-353 \mathrm{~K})$, and a modest dispersion $(\sigma=277 \mathrm{~K})$. This is expected because our method is purely spectroscopic, and the SSPP uses a combination of photometric and spectroscopic techniques. It has long been recognized that spectroscopically determined temperature estimates can be up to several hundred Kelvin cooler than photometric estimates. Regarding the $\log g$ estimate, a large systematic deviation exists for stars for which 
E. Fernández-Alvar et al.: Deep SDSS optical spectroscopy of distant halo stars. III.
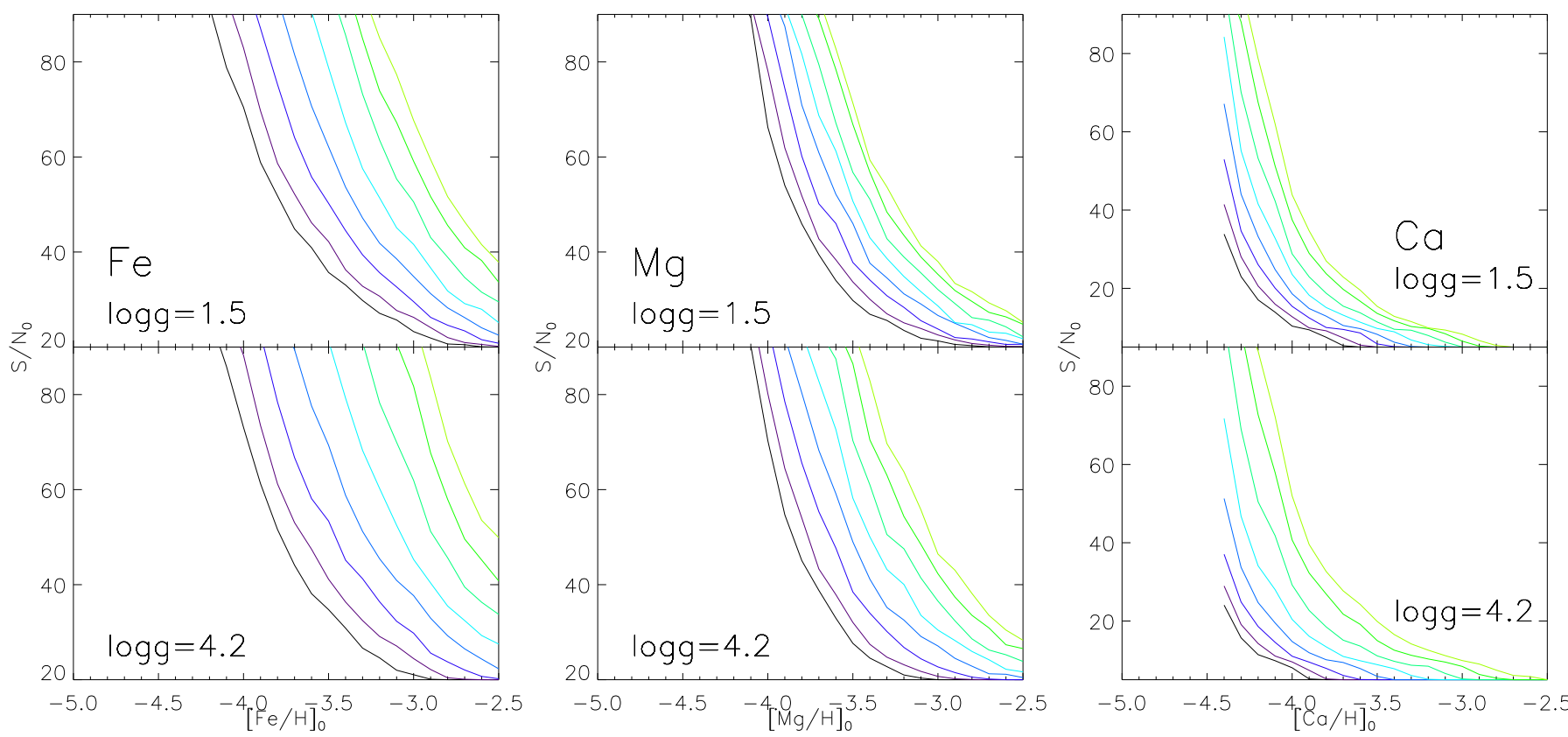

Fig. 3. Lowest required signal-to-noise values, $\mathrm{S} / \mathrm{N}_{0}$, to reliably determine $[\mathrm{Fe} / \mathrm{H}]_{0},[\mathrm{Mg} / \mathrm{H}]_{0}$ and $[\mathrm{Ca} / \mathrm{H}]_{0}$ abundances, evaluated at log $g=1.5$ and $\log g=4.5$ and $4750<T_{\text {eff }}<6500 \mathrm{~K}$ (darker colors correspond to lower temperatures). The increase in $T_{\text {eff }}$ complicates the [Fe/H] determination, mainly for $\mathrm{Fe}$ and $\mathrm{Mg}$, and a higher $\mathrm{S} / \mathrm{N}$ is necessary. The sensitivity of the $\mathrm{Ca} \mathrm{II} \mathrm{HK}$ resonance lines enables determining $[\mathrm{Ca} / \mathrm{H}]$ at very low abundances.

Table 2. Same as in Table 1, but for Mg.

\begin{tabular}{|c|c|c|c|}
\hline $\mathrm{Mg}$ & $\log g=1.5$ & & \\
\hline \multicolumn{4}{|c|}{$T_{\text {eff }}<5500 \mathrm{~K}$} \\
\hline$p_{1}$ & $1.205388 \mathrm{e}-04$ & $-1.318294 \mathrm{e}+00$ & $3.680773 e+03$ \\
\hline$p_{2}$ & $9.044162 \mathrm{e}-04$ & $-9.930854 \mathrm{e}+00$ & $2.772508 \mathrm{e}+04$ \\
\hline$p_{3}$ & $1.706439 \mathrm{e}-03$ & $-1.876632 \mathrm{e}+01$ & $5.232795 e+04$ \\
\hline \multicolumn{4}{|c|}{$T_{\text {eff }}>5500 \mathrm{~K}$} \\
\hline$p_{1}$ & $4.551823 \mathrm{e}-05$ & $-5.582935 \mathrm{e}-01$ & $1.766349 \mathrm{e}+03$ \\
\hline$p_{2}$ & $3.205082 \mathrm{e}-04$ & $-3.960549 \mathrm{e}+00$ & $1.252583 \mathrm{e}+04$ \\
\hline$p_{3}$ & $5.621326 \mathrm{e}-04$ & $-6.969943 \mathrm{e}+00$ & $2.201842 \mathrm{e}+04$ \\
\hline$l_{1}$ & $-2.316824 \mathrm{e}-03$ & $4.812897 \mathrm{e}+00$ & \\
\hline$l_{2}$ & $-1.307164 \mathrm{e}-03$ & $8.809123 \mathrm{e}+00$ & \\
\hline \multicolumn{4}{|c|}{$\log g=4.2$} \\
\hline \multicolumn{4}{|c|}{$T_{\text {eff }}<6000 \mathrm{~K}$} \\
\hline$p_{1}$ & $8.279952 \mathrm{e}-05$ & $-9.399753 \mathrm{e}-01$ & $2.729978 \mathrm{e}+03$ \\
\hline$p_{2}$ & $6.308861 \mathrm{e}-04$ & $-7.190066 \mathrm{e}+00$ & $2.083195 e+04$ \\
\hline$p_{3}$ & $1.210125 \mathrm{e}-03$ & $-1.378693 \mathrm{e}+01$ & $3.977522 \mathrm{e}+04$ \\
\hline \multicolumn{4}{|c|}{$T_{\text {eff }}>6000 \mathrm{~K}$} \\
\hline$p_{1}$ & $6.482425 \mathrm{e}-06$ & $-1.038303 \mathrm{e}-01$ & $4.460068 \mathrm{e}+02$ \\
\hline$p_{2}$ & $5.010227 \mathrm{e}-05$ & $-8.068283 \mathrm{e}-01$ & $3.330069 \mathrm{e}+03$ \\
\hline$p_{3}$ & $1.011057 \mathrm{e}-04$ & $-1.570228 \mathrm{e}+00$ & $6.187633 e+03$ \\
\hline$l_{1}$ & $-9.710505 \mathrm{e}-04$ & $-3.277127 \mathrm{e}+00$ & \\
\hline$l_{2}$ & $4.284561 \mathrm{e}-03$ & $-2.491109 \mathrm{e}+01$ & \\
\hline
\end{tabular}

we obtain surface gravity estimates of $\log g<3(\delta=-1.26 \mathrm{dex}$, $\sigma=1.56 \mathrm{dex})$. This is again not surprising because estimates of surface gravity are particularly challenging at low metallicity from low-S/N spectra. The $[\mathrm{Fe} / \mathrm{H}]$ comparison exhibits a relatively small negative offset $\delta=-0.16 \mathrm{dex}$, with a dispersion $\sigma=$ 0.33 dex. Essentially all of this offset can be accounted for by the differences in the temperature estimates.
Table 3. Same as in Table 1, but for Ca.

\begin{tabular}{|c|c|c|c|}
\hline $\mathrm{Ca}$ & $\log g=1.5$ & & \\
\hline \multicolumn{4}{|c|}{$T_{\text {eff }}<5500 \mathrm{~K}$} \\
\hline$p_{1}$ & $-3.711640 \mathrm{e}-04$ & $3.904669 \mathrm{e}+00$ & $-1.013811 \mathrm{e}+04$ \\
\hline$p_{2}$ & $-3.092381 \mathrm{e}-03$ & $3.245765 \mathrm{e}+01$ & $-8.415880 \mathrm{e}+04$ \\
\hline$p_{3}$ & $-6.420058 \mathrm{e}-03$ & $6.725487 \mathrm{e}+01$ & $-1.741799 \mathrm{e}+05$ \\
\hline \multicolumn{4}{|c|}{$T_{\text {eff }}>5500 \mathrm{~K}$} \\
\hline$p_{1}$ & $3.881031 \mathrm{e}-06$ & $-9.518567 \mathrm{e}-02$ & $5.899625 \mathrm{e}+02$ \\
\hline$p_{2}$ & $2.968447 \mathrm{e}-05$ & $-8.304656 \mathrm{e}-01$ & $5.092121 \mathrm{e}+03$ \\
\hline$p_{3}$ & $6.847159 \mathrm{e}-05$ & $-1.908617 \mathrm{e}+00$ & $1.119159 \mathrm{e}+04$ \\
\hline$l_{1}$ & $2.485399 \mathrm{e}-04$ & $-1.451917 \mathrm{e}+01$ & \\
\hline$l_{2}$ & $7.746718 \mathrm{e}-03$ & $-8.033748 \mathrm{e}+01$ & \\
\hline \multicolumn{4}{|c|}{$\log g=4.2$} \\
\hline \multicolumn{4}{|c|}{$T_{\text {eff }}<6000 \mathrm{~K}$} \\
\hline$p_{1}$ & $5.989651 \mathrm{e}-05$ & $-4.844238 \mathrm{e}-01$ & $9.931608 \mathrm{e}+02$ \\
\hline$p_{2}$ & $4.289678 \mathrm{e}-04$ & $-3.375793 e+00$ & $6.682556 \mathrm{e}+03$ \\
\hline$p_{3}$ & $7.663057 \mathrm{e}-04$ & $-5.832676 \mathrm{e}+00$ & $1.102958 \mathrm{e}+04$ \\
\hline \multicolumn{4}{|c|}{$T_{\text {eff }}>6000 \mathrm{~K}$} \\
\hline$p_{1}$ & $2.605201 \mathrm{e}-05$ & $-2.514639 \mathrm{e}-01$ & $6.919529 \mathrm{e}+02$ \\
\hline$p_{2}$ & $2.809412 \mathrm{e}-04$ & $-3.033114 \mathrm{e}+00$ & $8.964729 e+03$ \\
\hline$p_{3}$ & $7.024900 \mathrm{e}-04$ & $-8.000266 e+00$ & $2.432530 \mathrm{e}+04$ \\
\hline$l_{1}$ & $-1.835714 \mathrm{e}-03$ & $-1.504880 \mathrm{e}+00$ & \\
\hline$l_{2}$ & $4.354132 \mathrm{e}-03$ & $-5.797910 \mathrm{e}+01$ & \\
\hline
\end{tabular}

Finally, we compared the SSPP estimate of $[\alpha / \mathrm{Fe}]$ with our $[\mathrm{Ca} / \mathrm{Fe}]$ and $[\mathrm{Mg} / \mathrm{Fe}]$ measurements. Both show an offset $\delta \sim$ -0.15 dex and dispersion $\sigma \sim 0.26$ dex. Stars with the highest and lowest $[\mathrm{Ca} / \mathrm{Fe}]$ and $[\mathrm{Mg} / \mathrm{Fe}]$ estimates from our own estimates exhibit the largest differences.

To clarify whether the differences in the estimated stellar parameters $T_{\mathrm{eff}}$ and $\log g$ severely affect the resulting abundance 
measurements, we repeated the $[\mathrm{Fe} / \mathrm{H}],[\mathrm{Ca} / \mathrm{Fe}]$, and $[\mathrm{Mg} / \mathrm{Fe}]$ determinations with FERRE, but after replacing the stellar parameters with those from SSPP. The resulting offset in the $[\mathrm{Fe} / \mathrm{H}] \mathrm{de}-$ termination compares better with the SSPP $[\mathrm{Fe} / \mathrm{H}]$, although the dispersion increases slightly $(\delta=-0.09 \mathrm{dex}, \sigma=0.38 \mathrm{dex})$. Conversely, the dispersion in the comparison of the SSPP $[\alpha / \mathrm{Fe}]$ with the new $[\mathrm{Ca} / \mathrm{Fe}]$ and $[\mathrm{Mg} / \mathrm{Fe}]$ estimates increases by more than 0.2 dex, although the offset for $[\mathrm{Mg} / \mathrm{Fe}]$ is reduced from -0.14 dex to -0.01 dex. Thus, differences in $T_{\text {eff }}$ and $\log g$ are not solely responsible for the derived abundance contrast with the SSPP.

\subsection{Comparison with high-resolution analyses}

Several studies of EMP candidates from SDSS/SEGUE have been followed-up and analyzed with high-resolution spectra reported in the literature. Here we compare our present results with these measurements.

SDSS J031745.82+002304.1 was analyzed by Bonifacio et al. (2012) as one of their 16 EMP candidates found in the SDSS/SEGUE database. Our measurements for this star agree very well with their estimates from high-resolution spectra. We obtain $T_{\text {eff }}=5780 \mathrm{~K}, \log g=3.72,[\mathrm{Fe} / \mathrm{H}]>-3.40,[\mathrm{Ca} / \mathrm{Fe}] \geq$ +0.62 and $[\mathrm{Mg} / \mathrm{Fe}] \geq+0.21$, while they obtained $T_{\text {eff }}=5786 \mathrm{~K}$, $\log g=4.02,[\mathrm{Fe} / \mathrm{H}]=-3.46,[\mathrm{Ca} / \mathrm{Fe}]=+0.75(+0.60$ from $\mathrm{Ca}$ I lines) and $[\mathrm{Mg} / \mathrm{Fe}]=+0.38$.

Aoki et al. (2013) determined $T_{\text {eff }}, \log g,[\mathrm{Fe} / \mathrm{H}],[\mathrm{Ca} / \mathrm{Fe}]$, and $[\mathrm{Mg} / \mathrm{Fe}]$ for $70 \mathrm{VMP}$ and EMP stars selected from SDSS/SEQUE. Nine of these objects are in common with our sample: $[\mathrm{Fe} / \mathrm{H}]$ measurements from both analyses are available for seven stars, $[\mathrm{Ca} / \mathrm{Fe}]$ for five stars, and $[\mathrm{Mg} / \mathrm{Fe}]$ for six stars. Our $T_{\text {eff }}$ estimates are offset by $-241 \mathrm{~K}$ from the Aoki et al. results, with a dispersion $\sigma=169 \mathrm{~K}$. The $\log g$ comparison exhibits a larger offset and dispersion, $\delta=-1.03$ dex and $\sigma=1.21 \mathrm{dex}$, similar to the comparison obtained with respect to the SSPP results. The $[\mathrm{Fe} / \mathrm{H}]$ estimates compare reasonably well, with an offset of $\delta=-0.12 \mathrm{dex}$ and $\sigma=0.21 \mathrm{dex}$. The $[\mathrm{Mg} / \mathrm{Fe}]$ results are only in fair agreement, $\delta=-0.08$ dex and $\sigma=0.30$ dex. In contrast, our $[\mathrm{Ca} / \mathrm{Fe}]$ estimates are $0.43 \mathrm{dex}$ higher, with a dispersion of $\sigma=0.39$ dex. However, the Aoki et al. determination of $[\mathrm{Ca} / \mathrm{H}]$ in some of their stars came from the Ca I $4226 \AA$ equivalent width, which is more sensitive to non-LTE effects than the Ca II HK doublet we considered.

We identify SDSS J132250.59+012342.9 as an EMP star, as previously reported by Placco et al. (2015). For this star we obtained the following parameters: $T_{\text {eff }}=5234 \mathrm{~K}, \log g=0.84$, $[\mathrm{Fe} / \mathrm{H}]=-3.32,[\mathrm{Ca} / \mathrm{Fe}]=+0.23$ and $[\mathrm{Mg} / \mathrm{Fe}]=+0.30$. Placco et al. obtained a lower $T_{\text {eff }}=5008 \pm 100 \mathrm{~K}$, a higher $\log g=$ $1.95 \pm 0.20$, and a lower $[\mathrm{Fe} / \mathrm{H}]=-3.64 \pm 0.05$, which are in reasonable agreement with our estimates. They also obtained $[\mathrm{Ca} / \mathrm{Fe}]=+0.23 \pm 0.08$ and $[\mathrm{Mg} / \mathrm{Fe}]=+0.25 \pm 0.05$, which are also in good agreement with our results.

Recently, Susmitha Rani et al. (2016) reported $[\mathrm{Fe} / \mathrm{H}]=$ $-3.42 \pm 0.19$ for the star SDSS J134338.67+484426.6. This star is also included in our sample; we obtained estimates of $T_{\text {eff }}=5307 \mathrm{~K}, \log g=0.51,[\mathrm{Fe} / \mathrm{H}]<-3.7,[\mathrm{Ca} / \mathrm{H}]=-3.40$, and $[\mathrm{Mg} / \mathrm{H}]=-3.52$. Their temperature estimate is higher than ours, $T_{\text {eff }}=5620 \mathrm{~K}$, and their $\log g=3.44$ is considerably larger. We only determined an $[\mathrm{Fe} / \mathrm{H}]$ upper limit, which is lower than their $[\mathrm{Fe} / \mathrm{H}]$ measurement. They also determined $[\mathrm{Ca} / \mathrm{H}]=-3.23 \pm 0.16$, which is consistent with our estimate, and $[\mathrm{Mg} / \mathrm{H}]=-3.27 \pm 0.16$, slightly lower than our estimate.

In conclusion, the several comparisons we performed reveal that our temperature estimates are generally lower than previously reported results, by about $200-250 \mathrm{~K}$, which is due to our use of spectroscopic estimates, a significant underestimate in our determination of $\log g$, a slight underestimate of $[\mathrm{Fe} / \mathrm{H}]$ (understandable from the $T_{\text {eff }}$ offset), and a dispersion $\sim 0.3$ dex in the derived $[\mathrm{Ca} / \mathrm{Fe}]$ and $[\mathrm{Mg} / \mathrm{Fe}]$.

\section{Results}

Our final sample of EMP stars is listed in Table 4, along with their ugriz magnitudes and heliocentric velocities, calculated by the SSPP (the typical accuracy for these velocities is on the order of $\left.5 \mathrm{~km} \mathrm{~s}^{-1}\right)$. Tables 5 and 6 list our estimated stellar atmospheric parameters, $T_{\text {eff }}, \log g$, and $[\mathrm{M} / \mathrm{H}]$, and the derived abundances $[\mathrm{Fe} / \mathrm{H}],[\mathrm{Ca} / \mathrm{H}]$, and $[\mathrm{Mg} / \mathrm{H}]$, as well as their uncertainties. The minimum $\chi^{2}$ searches with FERRE were repeated ten times, with added random noise. The uncertainties are estimated from the standard deviation. We only list stars for which we obtained $-4.0<[\mathrm{Fe} / \mathrm{H}]<-3.0$. When our estimates were lower than the upper limit associated with the stellar parameters and $\mathrm{S} / \mathrm{N}$ of the spectrum from which it was measured, a corresponding upper limit is stated. We obtained $[\mathrm{Fe} / \mathrm{H}]$ measurements for 44 SDSS/SEGUE spectra and 4 BOSS spectra, $48 \%$ and $24 \%$ of each sample, respectively. For $[\mathrm{Mg} / \mathrm{H}]$, we determined reliable estimates for $86(93 \%)$ and $13(76 \%)$ of the stellar spectra in the SDSS/SEGUE and BOSS samples, respectively. The $[\mathrm{Ca} / \mathrm{H}]$ estimates were all reliable measurements. As noted above, $\mathrm{Fe}$ is the most difficult abundance to measure because its lines are so weak.

\section{1. $[\mathrm{Ca} / \mathrm{Fe}]$ and $[\mathrm{Mg} / \mathrm{Fe}]$}

The $\alpha$-element enrichment is of particular interest in this extremely low-metallicity regime, since it can provide valuable information on the nucleosynthesis histories of the first generations of stars. Figure 4 shows our derived $[\mathrm{Ca} / \mathrm{Fe}]$ and $[\mathrm{Mg} / \mathrm{Fe}]$ (split into SDSS/SEGUE and BOSS stars in the top and bottom panels) as a function of $[\mathrm{Fe} / \mathrm{H}]$. We use arrows to indicate upper limits on $[\mathrm{Fe} / \mathrm{H}]$ (hence lower limits on $[\mathrm{Ca}(\mathrm{Mg}) / \mathrm{Fe}])$. When neither $\mathrm{Ca}$ (or $\mathrm{Mg}$ ) nor $\mathrm{Fe}$ abundances were determined, we plot their ratio with an asterisk. Overall, it appears that most of the stars present a value of $[\mathrm{Ca}(\mathrm{Mg}) / \mathrm{Fe}]$ consistent with the expected halo-star value of $\sim+0.4$. From inspection of this figure, it is apparent that at the lowest metallicities, more stars lie above $[\alpha / \mathrm{Fe}]=+0.4$ than below this value .

From the stars for which we obtained reliable determinations of the abundances, we took into account results for which the deviation from $[\mathrm{Ca} / \mathrm{Fe}]=+0.4$ was higher than three times the uncertainties of the ratios over the metallicity, or the iron abundance, in each case. We found two stars that exhibit ratios of $[\mathrm{Ca} / \mathrm{Fe}]$ and $[\mathrm{Mg} / \mathrm{Fe}]$ significantly higher than +0.4 among the pre-BOSS stars: SDSS J035622.42+114705.4 $([\mathrm{Ca} / \mathrm{Fe}]=$ $+0.68 \pm 0.09 ;[\mathrm{Mg} / \mathrm{Fe}]=+0.87 \pm 0.11)$, and SDSS J031259.10$061957.1([\mathrm{Ca} / \mathrm{Fe}]=+0.89 \pm 0.10 ;[\mathrm{Mg} / \mathrm{Fe}]=+0.89 \pm 0.14)$. These stars are shown in Fig. 4 as green dots. However, we note that the SSPP estimates for these stars are $[\alpha / \mathrm{Fe}]=$ +0.14 and +0.41 , respectively. Hence, more accurate chemicalabundance estimates are required to confirm their status as $\alpha$-enhanced stars.

\section{2. $[\mathrm{C} / \mathrm{Fe}]$}

The SSPP provides $[\mathrm{C} / \mathrm{Fe}]$ measurements for 57 stars in our sample with detected carbon. After examining the resulting 
E. Fernández-Alvar et al.: Deep SDSS optical spectroscopy of distant halo stars. III.
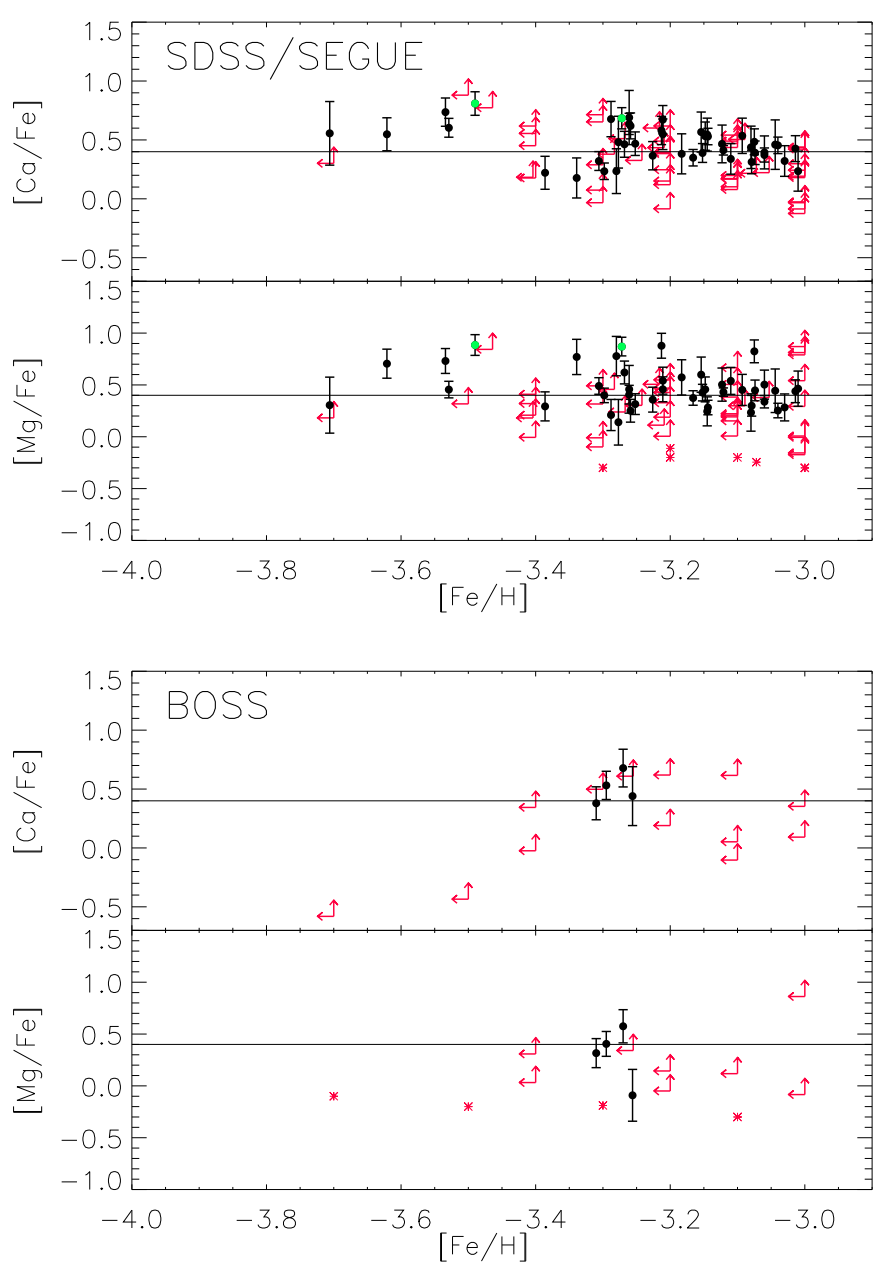

Fig. 4. $[\mathrm{Ca} / \mathrm{Fe}]$ and $[\mathrm{Mg} / \mathrm{Fe}]$ ratios as a function of $[\mathrm{Fe} / \mathrm{H}]$ from the analysis of our SDSS/SEGUE and BOSS samples. The reliable estimates after applying the upper limit evaluation are indicated as black dots. Red arrows show the cases where only an upper limit for the abundances could be obtained, and the direction the ratio would be situated in this diagram. The two stars for which we obtain high $[\mathrm{Ca} / \mathrm{Fe}]$ and $[\mathrm{Mg} / \mathrm{Fe}]$ are shown as green dots.

values, we find 28 carbon-enhanced metal-poor (CEMP) stars $([\mathrm{C} / \mathrm{Fe}]>+0.7)$, from which we obtain a cumulative frequency of CEMP stars below $[\mathrm{Fe} / \mathrm{H}]=-3.0$ of $\sim 26 \%$. We also verified that the frequency of CEMP stars increases as the metallicity decreases. Dividing our sample at the median metallicity for the stars with measured metallicity $([\mathrm{Fe} / \mathrm{H}]=-3.2)$, we find that $\sim 26 \%$ of the stars with $-3.2 \leq[\mathrm{Fe} / \mathrm{H}] \leq-3.0$ are CEMP stars, while $\sim 39 \%$ of the stars below $[\mathrm{Fe} / \mathrm{H}]=-3.2$ are CEMP stars. We also note that below a metallicity of -3.2 , the great majority of the stars shown in Fig. 5 are indeed CEMP stars, while the non-CEMP stars are the dominant fraction above this metallicity. Since more than half of our stars have only upper limits for $[\mathrm{Fe} / \mathrm{H}]$, we repeated the exercise with the SSPP estimates of $[\mathrm{Fe} / \mathrm{H}]$ for our full sample. For $[\mathrm{Fe} / \mathrm{H}] \leq-3.0$, we obtain a cumulative frequency of CEMP stars of $\sim 32 \%$; for $[\mathrm{Fe} / \mathrm{H}] \leq-3.5$, the cumulative frequency increases to $\sim 42 \%$. These results are consistent with the previous calculation within Poisson errors on the fractions (as a result of the small sample sizes involved, these are on the order of $10 \%$ ).

There is no evidence of a correlation between $[\mathrm{C} / \mathrm{Fe}]$ and $[\mathrm{Ca}(\mathrm{Mg}) / \mathrm{Fe}]$, nor with the SSPP $[\alpha / \mathrm{Fe}]$ estimates, as Fig. 6

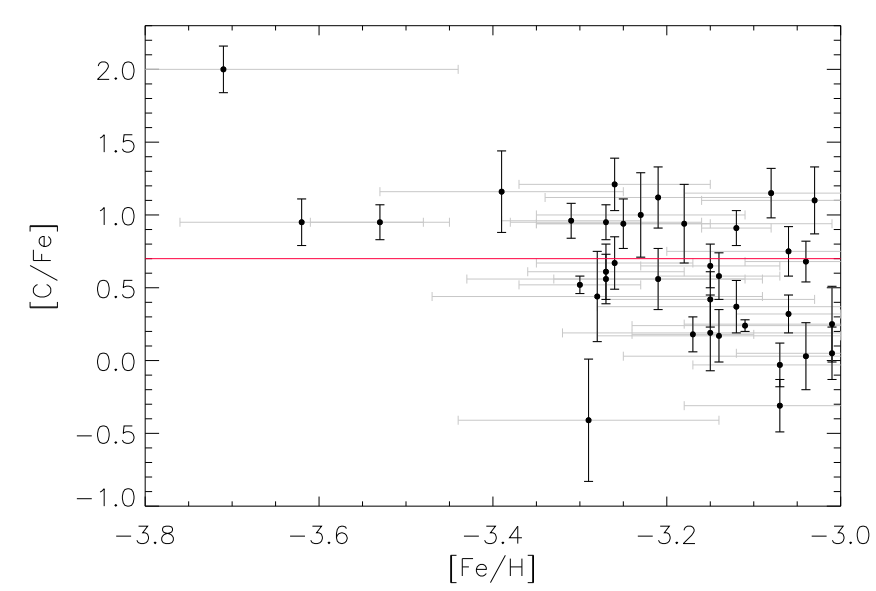

Fig. 5. $[\mathrm{C} / \mathrm{Fe}]$ estimates from the SSPP as a function of our $[\mathrm{Fe} / \mathrm{H}]$ estimate. The red line indicates the level of $[\mathrm{C} / \mathrm{Fe}]>+0.7$ used to evaluate whether a star is considered carbon enhanced.
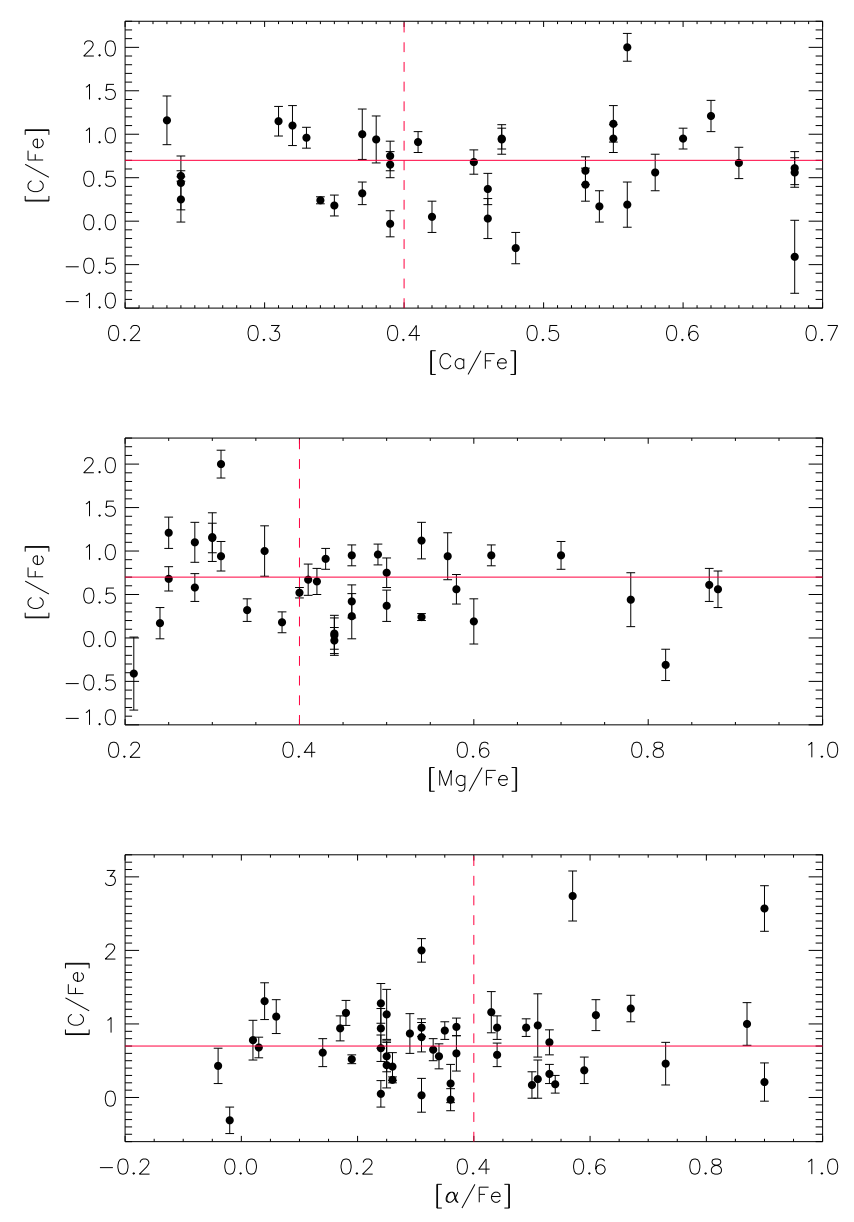

Fig. 6. $[\mathrm{C} / \mathrm{Fe}]$ estimates from the $\mathrm{SSPP}$ as a function of our $[\mathrm{Ca} / \mathrm{Fe}]$ and $[\mathrm{Mg} / \mathrm{Fe}]$ estimates (upper two panels, respectively). The lower panel shows $[\mathrm{C} / \mathrm{Fe}]$ as a function of the SSPP $[\alpha / \mathrm{Fe}]$ estimates. The red line indicates the level of $[\mathrm{C} / \mathrm{Fe}]>+0.7$ used to evaluate whether a star is considered carbon enhanced.

shows. Two stars exhibit high $[\mathrm{C} / \mathrm{Fe}](>+0.9)$ as well as $[\mathrm{Mg} / \mathrm{Fe}](>+0.6)$, but normal $[\mathrm{Ca} / \mathrm{Fe}]$ and $[\alpha / \mathrm{Fe}]$. 


\subsection{SDSS J134144.61+474128.6}

We call attention to the CEMP star SDSS J134144.61+474128.6 in our sample, which is a bright $(g=11.90)$ star with $[\mathrm{Fe} / \mathrm{H}]=$ -3.27 (the SSPP estimate of $[\mathrm{Fe} / \mathrm{H}]=-2.95$ ) and $[\mathrm{C} / \mathrm{Fe}]=$ +0.95 , identified during the course of the target search carried out for the MARVELS subsurvey (see the discussion of the MARVELS pre-survey in Rani et al. 2016). It is of interest that this star also exhibits elevated magnesium $([\mathrm{Mg} / \mathrm{Fe}]=+0.62)$, which is often found for CEMP-no stars (see, e.g., Norris et al. 2013). The absolute carbon abundance, $A(C)=\log \epsilon=6.11$, places it on the low- $C$ band that is associated with most CEMPno stars (see, e.g., Bonifacio et al. 2015; Yoon et al., in prep.). We note that the other bright EMP star in our sample, discussed by Rani et al. (2016; SDSS J134338.67+484426.6), is not carbon enhanced. Only 20 EMP stars have been identified to date that are as bright as these two stars, hence we plan to obtain a spectrum with higher resolution and higher $\mathrm{S} / \mathrm{N}$ of SDSS J1341+4741 in the near future.

\section{Conclusions}

We presented stellar atmospheric parameters and abundance estimates of $[\mathrm{Fe} / \mathrm{H}],[\mathrm{Mg} / \mathrm{H}]$, and $[\mathrm{Ca} / \mathrm{H}]$ for 108 extremely metalpoor stars from SDSS/SEGUE and BOSS with iron abundances in the range $-4.0<[\mathrm{Fe} / \mathrm{H}]<-3.0$. Below we summarize our main conclusions:

1. The determination of $\mathrm{Fe}$ and $\mathrm{Mg}$ abundances for EMP stars from individual lines in spectra at low spectral resolution $(R \sim 2000)$ depends critically on the signal-to-noise ratio because these lines are weak in this metallicity range. Conversely, the Ca II HK resonance lines in the blue region of the spectrum are sufficiently strong to reliably quantify the abundance of $\mathrm{Ca}$. We established a relation between $\mathrm{S} / \mathrm{N}$ and $[\mathrm{X} / \mathrm{H}]$ to determine upper limits on the abundances of $\mathrm{Fe}, \mathrm{Ca}$, and $\mathrm{Mg}$ when a reliable estimate was not possible.

2. We derived an analytical function that reproduced the detection limits for different elements as a function of $\mathrm{S} / \mathrm{N}$. These curves can be well fit by a parabola and a linear polynomial, with coefficients that depend on $T_{\text {eff }}$ and $\log g$. We reported analytical functions that specify the lowest required $\mathrm{S} / \mathrm{N}$ to reliably estimate $[\mathrm{Fe} / \mathrm{H}],[\mathrm{Ca} / \mathrm{H}]$, and $[\mathrm{Mg} / \mathrm{H}]$ in spectra with $R \sim 2000$ and $S / N<90$ from the spectral regions $4885<$ $\lambda<5070 \AA, 5220<\lambda<5280 \AA$, and $5295<\lambda<5500 \AA$ (to determine $\mathrm{Fe}$ ); $5160<\lambda<5190 \AA$ (to determine Mg abundances); and $3910<\lambda<3990 \AA$ (for Ca abundances).

3. We determined $[\mathrm{Ca} / \mathrm{Fe}]$ and $[\mathrm{Mg} / \mathrm{Fe}]$ abundance ratios for our program stars. The overall trend with metallicity is consistent with an $\alpha$-element enhancement $[\alpha / \mathrm{Fe}] \sim+0.4$ dex. However, several stars for which only Fe upper limits were estimated indicate high $[\alpha / \mathrm{Fe}]$ ratios, mainly at the lowest metallicities considered.

4. The $[\mathrm{C} / \mathrm{Fe}]$ estimates obtained with the SSPP revealed a cumulative frequency of $\sim 26 \%$ CEMP stars for $[\mathrm{Fe} / \mathrm{H}]<-3.0$, comparable to that found by Lee et al. (2013), 28\%. The frequency of CEMP stars also increases with decreasing metallicity, as reported by previous studies. We found no evidence for a $[\mathrm{C} / \mathrm{Fe}]$ correlation with $[\mathrm{Ca} / \mathrm{Fe}],[\mathrm{Mg} / \mathrm{Fe}]$, nor the SSPP $[\alpha / \mathrm{Fe}]$ measurements.

5. We identified a bright $(g=11.90)$ EMP star in our sample, SDSS J134144.61+474128.6, with enhanced [C/Fe] and $[\mathrm{Mg} / \mathrm{Fe}]$, as well as a low absolute carbon abundance, $A(C)=$ 6.11, a pattern typically associated with CEMP-no stars.
Higher resolution spectroscopic follow-up of this star is planned.

Several stars have reported $[\alpha / \mathrm{Fe}]$ significantly higher than +0.4 in the literature. For example, Aoki et al. (2007) reported on the highly $\alpha$-element enhanced VMP star from the HK survey, BS 16934-002, with $[\mathrm{Mg} / \mathrm{Fe}]=+1.23$, but normal $[\mathrm{Ca} / \mathrm{Fe}]=$ +0.44 , and no carbon overabundance. Other works reported high $[\mathrm{C} / \mathrm{Fe}]$ and $[\mathrm{Mg} / \mathrm{Fe}]$, but little evidence of $[\mathrm{Ca} / \mathrm{Fe}]$ enhancement with respect to the typical halo values (Norris et al. 2013; Yong et al. 2013; Hansen et al. 2015). Two scenarios were proposed for this chemical pattern: i) the results of mixing and processing of material due to stellar rotation; or ii) nucleosynthesis in mixing and fallback supernova models. Elements such as $\mathrm{Ca}$ and $\mathrm{Si}$ provide the key to understanding which of these possibilities is more likely. We only found two stars for which both [C/Fe] and $[\mathrm{Mg} / \mathrm{Fe}]$ are enhanced but that exhibit normal $[\mathrm{Ca} / \mathrm{Fe}]$, the expected chemical pattern for a massive spinstar (Norris et al. 2013; Maeder et al. 2015).

Puzia et al. (2006) measured $[\alpha / \mathrm{Fe}]$ ratios significantly higher than +0.5 for globular clusters in early-type elliptical galaxies based on Lick line-index measurements, at $-1<$ $[\mathrm{Z} / \mathrm{H}]<0-$ these authors used $[\mathrm{Z} / \mathrm{H}]$ to indicate the global metallicity in a galaxy, estimated from $\mathrm{Mg}$ and $\mathrm{Fe}$ lines (see Puzia et al. 2006; González 1993). They suggested that massive stars are the potential progenitors, with $M>20 M_{\odot}$, or with $M \sim$ 130-190 $M_{\odot}$ that explode as pair-instability SNe. Both possibilities imply extremely short timescales, on the order of few Myr. Therefore, the authors concluded that these stars may belong to the first generation of star clusters formed in their respective galaxies. In Paper II we reported high $[\mathrm{Ca} / \mathrm{Fe}]$ and $[\mathrm{Mg} / \mathrm{Fe}]$ median values for stars in the outer-halo region of the Galaxy, at Galactocentric radii greater than $40 \mathrm{kpc}$. Such stars could have been formed in low-mass fragments at very early stages of the evolution of the Milky Way, and later accreted into the Galactic halo (see, e.g., Tissera et al. 2014, and references therein).

In contrast, Caffau et al. (2013a) found three stars with $[\mathrm{Fe} / \mathrm{H}]<-3.0$ with low $[\alpha / \mathrm{Fe}]$ ratios. Similarly low $[\alpha / \mathrm{Fe}]$ stars had previously been detected by Nissen \& Schuster (2010). However, the latter authors found these ratios for stars with higher $[\mathrm{Fe} / \mathrm{H}]$, and interpreted this population as being born after the explosion of Type Ia SNe. In this scenario, the low $[\alpha / \mathrm{Fe}]$ ratios would then be the result of the addition of Fe from lowto intermediate-mass stars. However, at metallicities lower than $[\mathrm{Fe} / \mathrm{H}]=-3.0$, few SNIa explosions are expected to have occurred. The interpretation offered by Caffau et al. (2013a,b) is that two starbursts could have taken place in their progenitor fragment; the $[$ low $-\alpha / \mathrm{Fe}]$ ratios could then have resulted from gas enriched by SNIa explosions of stars formed in the first burst. This hypothesis was also invoked by Carigi et al. (2002) to explain low $[\mathrm{O} / \mathrm{Fe}]$ in Milky Way dwarf spheroidal satellites from chemical evolution models.

It would be desirable to obtain more accurate estimates to refine our reported $\alpha$-element enhancements in cases where the iron abundance could not be determined. The analysis performed in Paper II revealed high $[\alpha / \mathrm{Fe}]$ enhancements for very metalpoor stars in the outer-halo region of our Galaxy. Accurate $[\mathrm{Fe} / \mathrm{H}]$ estimates for our stars would allow us to test whether these results are confirmed by our new sample.

Acknowledgements. E.F.A. acknowledges support from DGAPA-UNAM postdoctoral fellowships. C.A.P. acknowledges support from the Spanish MINECO through grant AYA2014-56359-P. T.C.B. acknowledges partial support for this work from grants PHY 08-22648; Physics Frontier Center/Joint Institute or 
E. Fernández-Alvar et al.: Deep SDSS optical spectroscopy of distant halo stars. III.

Nuclear Astrophysics (JINA), and PHY 14-30152; Physics Frontier Center/JINA Center for the Evolution of the Elements (JINA-CEE), awarded by the US National Science Foundation. Y.S.L. acknowledges partial support from the National Research Foundation of Korea to the Center for Galaxy Evolution Research and Basic Science Research Program through the National Research Foundation of Korea (NRF) funded by the Ministry of Science, ICT \& Future Planning (NRF-2015R1C1A1A02036658). Funding for SDSS-III has been provided by the Alfred P. Sloan Foundation, the Participating Institutions, the National Science Foundation, and the U.S. Department of Energy Office of Science. The SDSS-III web site is http://www.sdss3.org/. SDSS-III is managed by the Astrophysical Research Consortium for the Participating Institutions of the SDSS-III Collaboration including the University of Arizona, the Brazilian Participation Group, Brookhaven National Laboratory, University of Cambridge, Carnegie Mellon University, University of Florida, the French Participation Group, the German Participation Group, Harvard University, the Instituto de Astrofisica de Canarias, the Michigan State/Notre Dame/JINA Participation Group, Johns Hopkins University, Lawrence Berkeley National Laboratory, Max Planck Institute for Astrophysics, Max Planck Institute for Extraterrestrial Physics, New Mexico State University, New York University, Ohio State University, Pennsylvania State University, University of Portsmouth, Princeton University, the Spanish Participation Group, University of Tokyo, University of Utah, Vanderbilt University, University of Virginia, University of Washington, and Yale University.

\section{References}

Alam, S., Albareti, F. D., Allende Prieto, C., et al. 2015, ApJS, 219, 12 Allende Prieto, C., Beers, T. C., Wilhelm, R., et al. 2006, ApJ, 636, 804 Allende Prieto, C., Sivarani, T., Beers, T. C., et al. 2008, AJ, 136, 2070 Allende Prieto, C., Fernández-Alvar, E., Schlesinger, K. J., et al. 2014, A\&A, 568, A7

Allende Prieto, C., Fernández-Alvar, E., Aguado, D. S., et al. 2015, A\&A, 579, A98

An, D., Beers, T. C., Johnson, J. A., et al. 2013, ApJ, 763, 65

An, D., Beers, T. C., Santucci, R. M., et al. 2015, ApJ, 813, L28

Aoki, W., Honda, S., Beers, T. C., et al. 2005, ApJ, 632, 611

Aoki, W., Honda, S., Beers, T. C. et al. 2007, ApJ, 660, 747

Aoki, W., Beers, T. C., Lee, Y. S., et al. 2013, AJ, 145, 13

Arnone, E., Ryan, S. G., Argast, et al. 2005, A\&A, 430, 507

Auer, L. 2003, Stellar Atmosphere Modeling, 288, 3

Barklem, P. S., Christlieb, N., Beers, T. C., et al. 2005, A\&A, 439, 129

Beers, T. C., Preston, G. W., \& Shectman, S. A. 1985, AJ, 90, 2089

Beers, T. C., Preston, G. W., \& Shectman, S. A. 1992, AJ, 103, 1987

Beers, T. C., Carollo, D., Ivezić, Ž., et al. 2012, ApJ, 746, 34

Bonifacio, P., Spite, M., Cayrel, R., et al. 2009, A\&A, 501, 519

Bonifacio, P., Sbordone, L., Caffau, E., et al. 2012, A\&A, 542, A87

Bonifacio, P., Caffau, E., Spite, M., et al. 2015, A\&A, 579, A28

Caffau, E., Bonifacio, P., François, P., et al. 2011a, Nature, 477, 67
Caffau, E., Bonifacio, P., François, P., et al. 2011b, A\&A, 534, A4 Caffau, E., Bonifacio, P., François, P., et al. 2012, A\&A, 542, A51 Caffau, E., Bonifacio, P., François, P., et al. 2013a, A\&A, 560, A15 Caffau, E., Bonifacio, P., Sbordone, L., et al. 2013b, A\&A, 560, A71 Carigi, L., Hernandez, X., \& Gilmore, G. 2002, MNRAS, 334, 117 Carollo, D., Beers, T. C., Lee, Y. S., et al. 2007, Nature, 450, 1020 Carollo, D., Beers, T. C., Chiba, M., et al. 2010, ApJ, 712, 692 Cayrel, R., Depagne, E., Spite, M., et al. 2004, A\&A, 416, 1117 Chen, Y. Q., Zhao,, G., Carrell, K., et al. 2014, ApJ, 795, 521 Christlieb, N. 2003, Rev. Mod. Phys., 16, 191

Cohen, J. G., Christlieb, N., McWilliam, A., et al. 2004, ApJ, 612, 1107 Cohen, J. G., McWilliam, A., Christlieb, N., et al. 2007, ApJ, 659, L161 Cohen, J. G., Christlieb, N., McWilliam, A., et al. 2008, ApJ, 672, 320 Dawson, K. S., Schlegel, D. J., Ahn, C. P., et al. 2013, AJ, 145, 10 de Jong, J. T. A., Yanny, B., Rix, H.-W., et al. 2010, ApJ, 714, 663 Eisenstein, D. J., Weinberg, D. H., Agol, E., et al. 2011, AJ, 142, 72 Fernández-Alvar, E., Allende Prieto, C., Schlesinger, K. J., et al. 2015, A\&A, 577, A81

Frebel, A., Aoki, W., Christlieb, N., et al. 2005, Nature, 434, 871

Frebel, A., Chiti, A., Ji, A. P., Jacobson, H. R., \& Placco, V. M. 2015, ApJ, 810, L27

González, J. J. 1993, Ph.D. Thesis, University of California, Santa Cruz Gunn, J. E., Siegmund, W. A., Mannery, E. J., et al. 2006, AJ, 131, 2332 Hansen, T., Hansen, C. J., Christlieb, N., et al. 2015, ApJ, 807, 173 Janesh, W., Morrison, H. L., Ma, Z., et al. 2016, ApJ, 816, 80 Keller, S. C., Bessell, M. S., Frebel, A., et al. 2014, Nature, 506, 463

Lai, D. K., Rockosi, C. M., Bolte, M., et al. 2009, ApJ, 697, L63 Lee, Y. S., Beers, T. C., Sivarani, T., et al. 2008a, AJ, 136, 2022 Lee, Y. S., Beers, T. C., Sivarani, T., et al. 2008b, AJ, 136, 2050 Lee, Y. S., Beers, T. C., Allende Prieto, C., et al. 2011, AJ, 141, 90 Lee, Y. S., Beers, T. C., Masseron, T., et al. 2013, AJ, 146, 132 Li, H., Aoki, W., Zhao, G., et al. 2015, PASJ, 67, 84

Maeder, A., Meynet, G., \& Chiappini, C. 2015, A\&A, 576, A56 Meléndez, J., Placco, V. M., Tucci-Maia, M., et al. 2016, A\&A, 585, L5 Nissen, P. E., \& Schuster, W. J. 2010, A\&A, 511, L10

Nissen, P. E., \& Schuster, W. J. 2011, A\&A, 530, A15

Norris, J. E., Bessell, M. S., Yong, D., et al. 2013, ApJ, 762, 25

Placco, V. M., Frebel, A., Lee, Y. S., et al. 2015, ApJ, 809, 136 Puzia, T. H., Kissler-Patig, M., \& Goudfrooij, P. 2006, ApJ, 648, 383 Reimers, D., \& Wisotzki, L. 1997, The Messenger, 88, 14

Roederer, I. U. 2009, AJ, 137, 272

Roederer, I. U., Schatz, H., Lawler, J. E., et al. 2014, ApJ, 791, 32

Smee, S. A., Gunn, J. E., Uomoto, A., et al. 2013, AJ, 146, 32

Smolinski, J. P., Lee, Y. S., Beers, T. C., et al. 2011, AJ, 141, 89

Susmitha Rani, A., Sivarani, T., Beers, T. C., et al. 2016, MNRAS, 458, 2648

Tissera, P., Beers, T. C., Carollo, D., et al. 2014, MNRAS, 439, 2128

Yanny, B., Rockosi, C., Newberg, H. J., et al. 2009, AJ, 137, 4377

Yong, D., Norris, J. E., Bessell, M. S., et al. 2013, ApJ, 762, 27

York, D. G., Adelman, J., Anderson, J. E., Jr., et al. 2000, AJ, 120, 1579 